\title{
Práticas de educação em saúde voltadas para função sexual feminina
}

\author{
Health education practices aimed at female sexual function: a systematic review \\ Prácticas de educación para la salud centradas en la función sexual femenina: \\ una revisión sistemática
}

Paholla Pinto dos Santos ${ }^{1 *}$, Anna Laura dos Santos Werneck ${ }^{1}$.. Iranete Corpes Oliveira França ${ }^{1}$, Marina Baia do Vale ${ }^{1}$, Mayra Herminia Simões Hamad Farias do Couto ${ }^{1}$, Claudia Jeane de Pontes Miranda ${ }^{1}$, Susanne Cristine Brito e Silva ${ }^{1}$.

\section{RESUMO}

Objetivo: Relatar uma revisão sistemática sobre a abordagem das práticas de Educação em Saúde voltadas para a Função Sexual Feminina. Métodos: A revisão sistemática utilizou como estratégia de busca às bases de dados Base de Dados em Evidências em Fisioterapia (PEDro), Biblioteca Nacional de Medicina dos Estados Unidos (pubMed), Biblioteca Eletrônica Científica Online (SciELO) e Biblioteca Virtual em Saúde (BVS); nos idiomas português, inglês e espanhol, no período de agosto a outubro de 2020, utilizando as palavras-chaves: "Educação em Saúde", "Mulheres", "Saúde Sexual" e "Sexualidade". Foram incluídos estudos publicados nos últimos 5 anos e ensaios clínicos randomizados que abordassem sobre as práticas de Educação em Saúde na Função Sexual de mulheres. Resultados: Dos 252 estudos selecionados inicialmente, apenas 5 preencheram os critérios de inclusão, descrevendo diferentes abordagens de práticas educativas de acordo com a necessidade do público-alvo de cada pesquisa. Considerações finais: abordagem de ações de Educação em Saúde voltadas para a Função Sexual de mulheres melhorou significativamente o funcionamento sexual, independentemente da característica ou fase da vida que a mulher esteja vivenciando.

Palavras-chave: Educação em saúde, Mulheres, Saúde sexual, Sexualidade.

\begin{abstract}
Objective: To carry out a systematic review on the approach of Health Education practices aimed at the Female Sexual Function. Methods: The search strategies were submitted to the Physiotherapy Evidence Database (PEDro), United States National Library of Medicine (pubMed), Electronic Scientific Online Library (SciELO) and Virtual Health Library (BVS); in portuguese, english and spanish, from august to october 2020, using the keywords: "Health Education", "Women", "Sexual Health" and "Sexuality". Studies published in the last 5 years, randomized clinical trials, that addressed the practices of Health Education in Sexual Function of women were included. Results: Of the 252 studies initially selected, only 5 met the inclusion criteria, describing different approaches to educational practices according to the needs of the target audience for each research. Final considerations: The approach of Health Education actions aimed at the Sexual Function of women significantly improved sexual functioning, regardless of the characteristic or phase of life that the woman is experiencing.
\end{abstract}

Keywords: Health education, Women, Sexual health, Sexuality.

1 Universidade da Amazônia (UNAMA), Belém - PA. *E-mail: paholla_santos@hotmail.com 


\section{RESUMEN}

Objetivo: Realizar una revisión sistemática de La acercarse de las prácticas de Educación en Salud dirigidas a la función sexual femenina. Métodos: Las estrategias de busque da se enviaron a las bases de datos Base de datos de evidencia de fisioterapia (PEDro), Biblioteca Nacional de Medicina de los Estados Unidos (pubMed), Biblioteca Científica Electrónica en Línea (SciELO) y Biblioteca Virtual de Salud (BVS); en portugués, inglés y español, entiempo agosto a octubre de 2020, utilizando las palabras clave: "Educación de La salud", "Mujeres", "Salud sexual" y "Sexualidad". Incluimos estudios publicados en los últimos 5años, pruebas clínicas aleatorizados abordonaría las prácticas de La Educación de La Salud en la Función Sexual de las mujeres. Resultados: De los 252 estudios seleccionados inicialmente, sólo5cumplieronconloscriterios de inclusión, describiendo diferentes enfoques de las prácticas educativas de acuerdo a las necesidades del público objetivo de cada investigación. Consideraciones finales: El enfoque de las acciones de Educación en Salud dirigidas a La Función Sexual de las mujeres mejoró significativamente el funcionamiento sexual, independientemente de la característica o etapa de la vida que la mujer estuve viviendo.

Palabras clave: Educación en salud, Mujeres, Salud sexual, Sexualidad.

\section{INTRODUÇÃO}

A sexualidade é um aspecto central na vida das pessoas e pode envolver o ato sexual, a orientação sexual, o erotismo, o prazer, a afetividade, o amor e a reprodução. Ela é vivenciada por meio de pensamentos, fantasias, desejos, crenças, atitudes, valores e relacionamentos. Nas sociedades, é alvo de normas morais, religiosas ou científicas, que são aprendidas desde a infância, envolvendo o nosso corpo, história, costumes, relações afetivas, aspectos biológicos, socioculturais e psicológicos (BRASIL, 2018).

A mulher vivencia diferentes etapas durante a vida, determinadas por mudanças hormonais, físicas, psicológicas e sociais, acarretando em diferenças fisiológicas e biológicas do funcionamento do corpo feminino, como as mudanças no ciclo reprodutivo, período gestacional, período do pós-parto e o climatério. Todas essas, influenciam diretamente na sexualidade feminina (CASTRO MR, et al.,2013).

A função sexual feminina é, sem dúvida, muito mais do que uma simples resposta biológica a um estímulo, representa um componente importante da saúde e qualidade de vida. A conquista e a manutenção da relação sexual adequada são fundamentais para a manifestação ou percepção do bem-estar (AMARAL WN, et al., 2012). Durante a década de 50, os autores Masters e Johnson iniciaram uma minuciosa investigação científica acerca do comportamento sexual, apresentando uma descrição da resposta sexual humana que foi dividida em 4 fases sequenciais: excitação, platô, orgasmo e resolução (MASTERS WH e JOHNSON VE, 1984).

No final da década de 70, Kaplan propôs um modelo alternativo da resposta sexual, abrangendo o desejo como uma fase para o início do ato sexual. Esse modelo consiste em 3 fases: desejo, excitação e orgasmo (KAPLAN HS, 1977). Com a associação entre os modelos de Masters e Johnson e de Kaplan foram determinados critérios diagnósticos para os transtornos da sexualidade que constam no Manual Diagnóstico e Estatístico dos Transtornos Mentais (DSM), que estabeleceu a resposta sexual como um conjunto de 4 etapas sucessivas: desejo, excitação, orgasmo e resolução (DSM-IV, 2002).

O desejo é uma sensação que gera vontade de ter relação sexual proporcionando bem-estar físico e mental em relação ao sexo. A fase de excitação é a percepção de prazer na vulva e vagina, ocorrendo o intumescimento da genitália pelo aumento do aporte sanguíneo para essa região, que fica úmida pela lubrificação vaginal (ABDO CHN, et al., 2019). O orgasmo caracteriza-se pelo clímax de prazer sexual, que ocorre após uma fase de crescente excitação. $\mathrm{Na}$ fase de resolução, o organismo retorna as condições físicas e emocionais habituais, levando a sensação de relaxamento geral. Qualquer alteração persistente ou recorrente em uma dessas fases é denominada de disfunção sexual feminina (BRASIL, 2013). 
As Disfunções Sexuais Femininas (DSFs) são consideradas pela Organização Mundial da Saúde (OMS) um problema de saúde pública, influenciando diretamente na vida social, psicológica, ocupacional e física das mulheres e de seus parceiros, cujo DSM-IV, no capítulo denominado Transtornos Sexuais e da Identidade de Gênero compreende múltiplas desordens (DSM-IV, 2002). A partir do DSM-IV, foi criado um novo manual, intitulado DSM-V, que ampliou a classificação das DSFs como um grupo de transtornos heterogêneos caracterizados por uma perturbação na capacidade para responder sexualmente ou de sentir prazer sexual (ARAÚJJO AC e NETO FL, 2014).

Diante da nova perspectiva sobre o funcionamento sexual feminino e a compreensão das desordens, a OMS preconiza que a saúde sexual não seja considerada apenas a inexistência de disfunções, doenças ou debilidades e sim um estado físico, emocional, mental e social de bem-estar. Dessa forma, é essencial que essa temática seja desenvolvida na comunidade através de ações, com o intuito de colaborar e melhorar a saúde e qualidade de vida das pessoas (BRASIL, 2013).

As alterações da função sexual são multifatoriais, necessitando de uma abordagem holística, sendo necessário um tratamento biopsicossocial, multidisciplinar, associando medidas farmacológicas, fisioterapêuticas e intervenções psicológicas. Normalmente, é uma mistura entre métodos cognitivocomportamental e intervenções sistêmicas, incluindo educação sexual, permissão sexual, treinamento de comunicação, aconselhamento de casais, exercícios de conscientização física e da sensualidade (ARAÚJO JSA, et al., 2018).

A educação está presente na vida do ser humano, ela prevê interação entre as pessoas envolvidas dentro do contexto educativo e destas com o mundo. O termo Educação em Saúde indica uma correlação entre as duas áreas: a educação, caracterizando-se por métodos pedagógicos para transformar comportamentos, e a saúde, baseando-se nos conhecimentos científicos capazes de intervir sobre determinada patologia (FALKENBERG MB, et al., 2014).

Sendo assim, a educação em saúde é uma ferramenta de extrema importância na produção do cuidado e um indutor de práticas que promovam a criação de vínculo e o protagonismo do usuário diante do processo saúde-doença. Além disso, observam-se os determinantes e condicionantes de saúde para planejar e executar as ações. Essas estratégias são de suma importância em todos os níveis de atenção, mas, em especial na atenção básica que tem como característica a relação com a população de seu território (GOMES NMC, et al., 2019).

Portanto, abordar a educação em saúde voltada para a Função Sexual é oferecer informações sobre o entendimento do funcionamento, ciclo da resposta sexual feminina e possíveis disfunções. Trata-se de esclarecer que a sexualidade é construída ao longo de toda a vida, por meio da compreensão da afetividade e da busca por interações emocionais e físicas. Essas ações podem ser desenvolvidas em forma de palestras, divulgação de panfletos ilustrados com os componentes da anatomia feminina e abordagens sobre autoconhecimento (ABDO CHN, et al., 2019).

As atividades educativas podem e devem ser desenvolvidas nos serviços de saúde e nos diversos espaços sociais existentes na comunidade, promovendo a participação de forma coletiva, dando oportunidade das mulheres compartilharem suas experiências e aprendizados acerca da função sexual (BRASIL, 2013). Diante disso, o objetivo deste estudo é realizar uma revisão sistemática sobre a abordagem das práticas de Educação em Saúde voltadas para a Função Sexual Feminina.

\section{MÉTODOS}

O planejamento para esta revisão foi baseado nas orientações dos Principais Itens para Relatar Revisões Sistemáticas e Meta-análises (PRISMA), uma diretriz criada por Moheret D, et al. (2009) e atualizada recentemente por Vrabel M (2015), que consiste em um checklist de 27 itens e um fluxograma que tem por objetivo auxiliar os autores na elaboração de uma revisão sistemática ou meta-análise. 
Este estudo analisou artigos científicos referentes ao tema relacionado à abordagem das práticas de Educação em Saúde voltadas para Função Sexual de mulheres. A análise dos artigos foi realizada por duas pesquisadoras de forma independente, a fim de selecionarem os estudos potencialmente relevantes para a revisão.

A busca foi executada de forma online nas bases de dados: PEDro, PubMed, SciELO e BVS. A pesquisa ocorreu no período de agosto a outubro de 2020, utilizando os Descritores em Ciências da Saúde (DeCS), oficial da Biblioteca Virtual em Saúde, em português, inglês e espanhol respectivamente: "educação em saúde; mulheres; saúde sexual e sexualidade", e seus correlatos "health education; women; sexual health; sexuality"; "educación em salud; mujeres; salud sexual e sexualidad" com os operadores boleanos "AND" e "OR".

Nas bases de dados foram realizados os seguintes cruzamentos de descritores: "educação em saúde AND mulheres AND saúde sexual OR sexualidade; health education AND women AND sexual health OR sexuality; educación em salud AND mujeres AND salud sexual OR sexualidad".

$\mathrm{Na}$ plataforma PEDro, foi utilizado o seguinte filtro: clinical trial, sendo identificados 5 artigos. $\mathrm{Na}$ pubMed, foram encontrados 121 estudos com os filtros: 5 years, female, humans e clinical trial. Na SciELO foram observados 90 artigos apenas com o filtro de +2016. Já na BVS foram localizados 36 artigos com os seguintes filtros: educação em saúde, saúde sexual, educação sexual, ensaios clínicos, 5 anos, idiomas português, inglês e espanhol.

Para iniciar a seleção dos estudos, foi efetuada a leitura do título e resumo quando estes não relatavam informações suficientes sobre os critérios de inclusão e exclusão, porém tinham relação com a temática, era realizada a leitura do texto na íntegra. Em seguida, foram selecionados os artigos a partir dos critérios de elegibilidade.

A pergunta norteadora da pesquisa foi baseada segundo os componentes: População, Intervenção, Comparação e "Outcomes" (PICO), recomendado pelas Diretrizes Metodológicas do Ministério da Saúde, 2012, adaptado do PRISMA. Para o estudo foi utilizado à população com mulheres a partir de 18 anos, a Intervenção com práticas de Educação em Saúde, Comparação da Função Sexual e desfechos dos resultados apresentados (Quadro 1).

Foram considerados para análise somente ensaios clínicos randomizados que tivessem como públicoalvo mulheres a partir de 18 anos, publicados nos últimos 5 anos, nos idiomas português, inglês e espanhol, que abordassem sobre as práticas de Educação em Saúde voltadas para a Função Sexual de mulheres. Foram excluídos estudos de Revisão Sistemática de baixa evidência científica (escore menor ou igual a 3 na escala Jadad), que incluíssem apenas homens, mulheres com Incontinência Urinária ou com alteração cognitiva, como também estudos que desenvolvessem apenas intervenções psicoeducacionais.

A qualidade dos artigos elegíveis foi avaliada pela escala Jadad, utilizando pontuações estabelecidas de acordo com os critérios metodológicos adotados, contendo 5 itens e pontuação máxima de 5 , a fim de avaliar a qualidade metodológica dos artigos, analisar os dados rigorosamente, reduzir o risco de vieses, fornecendo assim a melhor evidência científica.

Foi realizada uma análise baseada no QUALIS/CAPES com a finalidade de avaliar a qualidade dos periódicos científicos e também, o Fator de Impacto da revista, capaz de avaliar a relevância dos estudos científicos, calculando o número médio de citações dos artigos publicados (Quadro 2).

\section{RESULTADOS}

Os artigos foram analisados nas seguintes fases: identificação, seleção, elegibilidade e inclusão. Na identificação, foram observados 252 através da busca nas bases de dados, destes, 5 foram excluídos por serem duplicados, restando 247. Ao aplicar os critérios de elegibilidade, 21 foram selecionados para leitura dos resumos, posteriormente, restaram 15 para serem lidos na íntegra e avaliados na escala Jadad, dentre os quais 5 foram incluídos na presente revisão de acordo com o fluxograma (Figura 1). 
Figura 1 - Fluxograma dos artigos avaliados.

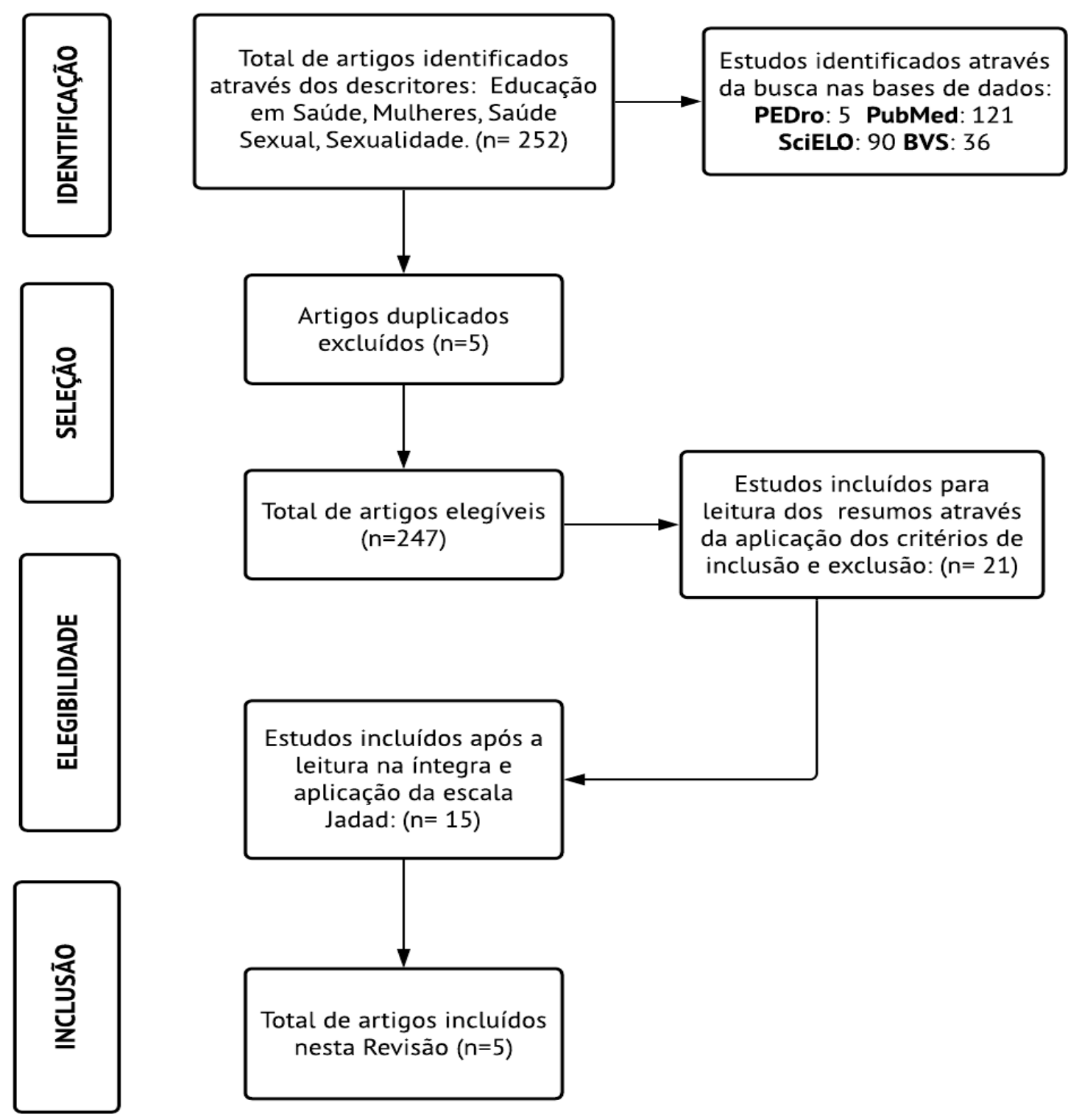

Fonte: Santos PP, et al., 2020.

Os trabalhos que foram incluídos descreviam diferentes abordagens acerca da Educação em Saúde voltada para Função Sexual, sendo realizado, em todos, a avaliação através do questionário do Índice de Função Sexual Feminina (FSFI) que contém 19 itens abrangendo 6 domínios: desejo, excitação, lubrificação, orgasmo, satisfação e dor.

Já nas intervenções foram disponibilizados materiais educativos, palestras, apresentações de slides, fotos e folders de acordo com a necessidade do público-alvo de cada pesquisa (mulheres histerectomizadas, gestantes e na pós-menopausa), além da didática de perguntas e respostas, bem como treinamento dos músculos do assoalho pélvico (exercícios de Kegel), ambas direcionadas para função sexual (Quadro 1). 
Quadro 1 - Características dos estudos incluídos nesta revisão sistemática.

\begin{tabular}{|c|c|c|c|}
\hline Autor/Ano & Público-alvo & Intervencão & fechos \\
\hline $\begin{array}{l}\text { Alamdari A, } \\
\text { et al., 2016. }\end{array}$ & $\begin{array}{l}48 \text { Mulheres pós histerectomia. } \\
\text { Grupo intervenção (GI): programa educacional } \\
\text { modelo PRECEDE ( } n=24) ; \\
\text { Idade não relatada, apenas a média }(48,12 \pm 3,61) \\
\text { Grupo controle }(G C) \text { : intervenções de rotina }(n=24) \text {; } \\
\text { Média idade }(3,72 \pm 48,54) \text {. }\end{array}$ & $\begin{array}{l}\text { Foram realizadas } 5 \text { sessões de } 45-60 \text { minutos cada, } 1 \text { vez por } \\
\text { semana, baseadas no modelo Predisponente, Reforçado, Capacitador } \\
\text { em Diagnóstico e Avaliação Educacional (PRECEDE). } \\
\text { GI: palestras, períodos de perguntas e respostas (tópicos incluindo } \\
\text { histerectomia, problemas sexuais, atitudes sexuais, estratégias e } \\
\text { habilidades para melhorar função sexual), apresentações de slides. } \\
\text { GC: intervenções de rotina e treinamento sobre cuidados pós } \\
\text { histerectomia. }\end{array}$ & $\begin{array}{l}\text { Efeito positivo do treinamento baseado no } \\
\text { modelo PRECEDE na função sexual de } \\
\text { mulheres histerectomizadas, indicando que a } \\
\text { educação sexual pode ser desenhada com } \\
\text { esse modelo. }\end{array}$ \\
\hline $\begin{array}{l}\text { Alavi MA, et } \\
\text { al., 2016. }\end{array}$ & $\begin{array}{l}98 \text { Mulheres na pós-menopausa. } \\
\text { Idade: } 40-60 \text { anos. } \\
\text { Gl: programa de aprimoramento sexual }(n=48) \text {. } \\
\text { GC: material educacional sobre a pós-menopausa } \\
(n=50) \text {. }\end{array}$ & $\begin{array}{l}\text { Foram realizadas sessões durante } 12 \text { semanas com duração de } 2 \\
\text { horas. } \\
\text { GI: recebeu um programa de aprimoramento sexual, incluindo sessões } \\
\text { educacionais em grupo, sobre vários aspectos da função sexual, } \\
\text { correção de atitudes em relação ao sexo e introdução de lubrificantes } \\
\text { para alívio da dispareunia. } \\
\text { GC: material educativo geral relacionado à menopausa, abordando } \\
\text { mudanças fisiológicas e psicológicas, bem como as formas de lidar } \\
\text { com as mesmas. }\end{array}$ & $\begin{array}{l}\text { O programa de aprimoramento sexual foi eficaz } \\
\text { em melhorar a função sexual de mulheres na } \\
\text { pós-menopausa, sendor necessária } \\
\text { aimplementação de programas educacionais } \\
\text { que levem em consideração o contexto cultural } \\
\text { do respectivo público. }\end{array}$ \\
\hline $\begin{array}{l}\text { Alavi MA, et } \\
\text { al., } 2017 .\end{array}$ & $\begin{array}{l}145 \text { Mulheres na pós-menopausa. } \\
\text { Idade: } 40-60 \text { anos. } \\
\text { GI 1: educação sexual formal }(n=47) \text {. } \\
\text { GI 2: exerćícios de Kegel }(n=48) \text {. } \\
\text { GC: cuidados de rotina na pós- menopausa }(n=50) \text {. }\end{array}$ & $\begin{array}{l}\text { GI 1: foram submetidas a um programa formal de educação sexual. } \\
\text { GI 2: foram submetidas a treinamento dos músculos do assoalho } \\
\text { pélvico (exercícios de Kegel). } \\
\text { GC: receberam material educativo geral sobre o climatério. } \\
\text { Os } 3 \text { grupos foram convidados a participarem de suas respectivas } \\
\text { sessões educacionais, cada um com data específica, sendo grupos de } \\
10 \text { a } 15 \text { membros, por } 2 \text { horas, usando apresentações e vídeos em } \\
\text { PowerPoint. Além disso, todos receberam livretos educacionais } \\
\text { referentes a sessões que estavam participando. }\end{array}$ & $\begin{array}{l}\text { Os programas de educação sexual e exercícios } \\
\text { de Kegel mostram-se eficazes na melhora de } \\
\text { alguns domínios da função sexual (excitação, } \\
\text { orgasmo e satisfação) em mulheres na pós- } \\
\text { menopausa. }\end{array}$ \\
\hline $\begin{array}{c}\text { Firouzi A, et } \\
\text { al., } 2019 .\end{array}$ & $\begin{array}{l}46 \text { Mulheres na pós-menopausa. } \\
\text { Idade: } 44-55 \text { anos. } \\
\text { Gl: programa educacional de aconselhamento } \\
\text { baseado em parteiras }(n=23) \text {. } \\
\text { GC: cuidados de rotina para pós-menopausa }(n=23) \text {. }\end{array}$ & $\begin{array}{l}\text { Sessões realizadas individualmente, incluindo } 4 \text { sessões de } 70 \\
\text { minutos, realizadas em intervalos de } 10 \text { dias. } \\
\text { GI: aconselhamento educacional baseado em parteiras sobre } \\
\text { menopausa, alterações fisiológicas e anatômicas, função e disfunção } \\
\text { sexual que afetam a mulher durante a menopausa. } \\
\text { GC: receberam apenas cuidados de rotina para meia-idade, diabetes, } \\
\text { pressão arterial, dislipidemia e rastreamento do câncer cervical e de } \\
\text { mama. }\end{array}$ & $\begin{array}{l}\text { O programa educacional de aconselhamento } \\
\text { baseado em parteiras melhorou } \\
\text { significativamente a função sexual em mulheres } \\
\text { na pós- menopausa. A literatura deixa claro que } \\
\text { intervenções de aconselhamento e educação } \\
\text { devem ser promovidas para ajudar a mulher a } \\
\text { superar possíveis problemas em seus } \\
\text { relacionamentos sexuais. }\end{array}$ \\
\hline $\begin{array}{l}\text { Mahnaz E, } \\
\text { et al., } 2020\end{array}$ & $\begin{array}{l}70 \text { Mulheres grávidas de } 4 \text { centros de saúde. } \\
\text { Idade: } 18-45 \text { anos. } \\
\text { GI: pacote educacional estruturado }(n=36) \text {. } \\
\text { GC: treinamento sobre amamentação e parto normal } \\
(n=34) \text {. }\end{array}$ & $\begin{array}{l}4 \text { sessões com aproximadamente } 10 \text { gestantes em cada centro de } \\
\text { saúde. } \\
\text { Durante as sessões eram fornecidas às gestantes fotos, pôsteres, } \\
\text { livretos educativos sobre as mudanças fisiológicas durante a gravidez e } \\
\text { saúde sexual de modo geral. } \\
\text { GI: recebeu pacote educacional estruturado desenvolvido com base na } \\
\text { Análise, Desenho, Desenvolvimento, Implementação e Avaliação } \\
\text { (ADDIE). } \\
\text { GC: treinamento sobre amamentação e parto normal. }\end{array}$ & $\begin{array}{l}\text { O pacote educacional estruturado teve um } \\
\text { efeito positivo sobre a pontuação total e } \\
\text { 5domínios do FSFI (desejo, excitação, } \\
\text { orgasmo, satisfação e lubrificação), além de } \\
\text { reduzir a disfunção sexual em gestantes, } \\
\text { aumentando o conhecimento e mudando as } \\
\text { atitudes em relação ao comportamento sexual } \\
\text { na gravidez. }\end{array}$ \\
\hline
\end{tabular}

Legenda: N: número de participantes; Gl: grupo intervenção; GC: grupo controle; Gl 1: grupo intervenção 1; Gl 2: grupo intervenção 2. Fonte: Santos PP, et al., 2020. 
Por fim, a qualidade dos artigos foi avaliada pela escala Jadad no qual todos os estudos obtiveram pontuação máxima correspondente a um escore de 5. A análise baseada no QUALIS/CAPES com a finalidade de avaliar a qualidade dos periódicos científicos variou entre B1 e A2, estando a maioria no estrato superior de qualidade de produção científica e também, o Fator de Impacto da revista, capaz de avaliar a relevância dos estudos científicos, calculando o número médio de citações dos artigos publicados, variando entre 2.216 a 3.800 (Quadro 2).

Quadro 2 - Avaliação dos artigos através da escala de Jadad e das revistas através do Qualis/CAPES e Fator de impacto.

\begin{tabular}{|c|c|c|c|c|c|}
\hline Autores/ Ano & $\begin{array}{c}\text { Alamdari A, } \\
\text { et al., 2016. }\end{array}$ & $\begin{array}{c}\text { Alavi MA, } \\
\text { et al., 2016. }\end{array}$ & $\begin{array}{c}\text { Alavi MA, } \\
\text { et al., 2017. }\end{array}$ & $\begin{array}{c}\text { Firouzi A, et } \\
\text { al., 2019. }\end{array}$ & $\begin{array}{c}\text { Mahnaz E, } \\
\text { et al., 2020 }\end{array}$ \\
\hline $\begin{array}{c}\text { 1- O estudo foi descrito } \\
\text { como randomizado? }\end{array}$ & 1 & 1 & 1 & 1 & 1 \\
\hline $\begin{array}{c}\text { 2- A randomização foi } \\
\text { descrita e adequada? }\end{array}$ & 1 & 1 & 1 & 1 & 1 \\
\hline $\begin{array}{c}\text { 3- Houve comparações e } \\
\text { resultados? }\end{array}$ & 1 & 1 & 1 & 1 & 1 \\
\hline $\begin{array}{c}\text { 4- As comparações e } \\
\text { resultados foram descritos } \\
\text { e são adequados? }\end{array}$ & 1 & 1 & 1 & 1 & 1 \\
\hline $\begin{array}{c}\text { 5- Foram descritas as } \\
\text { perdas e exclusões? }\end{array}$ & 1 & 1 & 1 & 1 & 1 \\
\hline Pontuação & $5 / 5$ & $5 / 5$ & $5 / 5$ & $5 / 5$ & $5 / 5$ \\
\hline QUALIS/CAPES & $\mathrm{A} 3$ & $\mathrm{~A} 2$ & $\mathrm{~B} 1$ & - & $\mathrm{A} 3$ \\
\hline Fator de impacto & 2.216 & 2.566 & 3.800 & 2.942 & 2.216 \\
\hline
\end{tabular}

Legenda: 1: Sim; 0: Não. Fonte: Santos PP, et al., 2020.

\section{DISCUSSÃO}

Os estudos utilizaram o questionário FSFI que contém 19 itens e 6 domínios (desejo, excitação, orgasmo, lubrificação, dor e satisfação), sendo classificados numa escala de 0 a 6 , determinando que escores mais altos indicam melhor função sexual. Além disso, foram utilizados outros questionários para obter dados socioeconômicos e demográficos.

A principal característica da Educação em Saúde é instituir ações que abordem de forma clara o tema proposto visando à promoção da saúde, pois estimula um processo de diálogo, indagação, reflexão, questionamento e ação partilhada. Tratando-se de Educação Sexual, é importante a compreensão das necessidades da população, a fim de solucionar suas possíveis queixas e condições de saúde, objetivando oferecer às pessoas os conhecimentos acerca da sexualidade e reprodução (BRASIL, 2013).

No presente estudo foram observadas formas de desenvolver as ações educacionais respeitando as peculiaridades da população estudada. Foram utilizadas como intervenções: informações sobre anatomia e fisiologia da mulher, palestras referentes à saúde sexual, apresentação de slides, distribuição de materiais educativos, treinamento dos músculos do assoalho pélvico, além de esclarecimentos sobre cuidados gerais.

Em alguns estudos, não foram descritos detalhadamente critérios importantes relacionados à aplicação da intervenção, como a idade, número e tempo de sessões. Cada autor selecionou uma população específica para realizar sua pesquisa. Alamdari A, et al. (2016) descreveram um programa voltado para mulheres com histerectomia, já Alavi MA, et al. (2016) e Alavi MA, et al. (2017) e Firouzi A, et al. (2019) realizaram práticas para mulheres na pós-menopausa e Mahnaz E, et al. (2020) utilizaram um pacote educacional para mulheres durante a gravidez. 
Alamdari A, et al. (2016) direcionaram seu estudo para um modelo teórico eficaz para identificar necessidades em educação e promoção da saúde que consiste em duas partes: Predisponente, Reforçado, Capacitador em Diagnóstico e Avaliação Educacional (PRECEDE) e Construção de Políticas, Regulatórias, Organizacionais em Desenvolvimento Educacional e Ambiental (PROCEED). Apenas a parte PRECEDE foi aplicada, a qual fornece uma estrutura em que fatores predisponentes (conhecimento, atitudes e crenças), fatores de reforço (influência da família, parceiros e profissionais da saúde) e fatores facilitadores (recursos e habilidades) podem influenciar no comportamento.

Dessa forma, Alamdari A, et al. (2016) utilizaram no grupo intervenção um programa educacional que consistia em 5 sessões de 45 a 60 minutos cada, 1 vez por semana. Foram aplicados métodos de ensino que incluíam palestras, períodos de perguntas e respostas, apresentação de slides e distribuição de folhetos educativos, todos com tópicos incluindo histerectomia, problemas e atitudes sexuais pós histerectomia, estratégias e habilidades para melhorar a função sexual. O grupo controle recebeu apenas intervenções de rotina e treinamento que cobriu cuidados pós histerectomia, mas não educação sexual. Tal programa educacional obteve efeitos positivos em termo de função sexual de mulheres pós histerectomia, sobretudo no grupo intervenção, no qual houve aumento tanto no escore total do FSFI quanto nos seus domínios.

De acordo com Alavi MA, et al. (2016), no estudo com mulheres na pós-menopausa, para o grupo intervenção, foram realizadas sessões educacionais de aprimoramento sexual, abordando aspectos da função sexual, a correção de atitudes em relação ao sexo durante a menopausa e a introdução de lubrificantes para aliviar a dispareunia devido à secura vaginal. Foram acompanhadas semanalmente, por telefone, possibilitando resolução de dúvidas. O grupo controle recebeu material educativo, fornecendo informações gerais sobre mudanças fisiológicas e psicológicas durante o período da menopausa. Os grupos foram acompanhados em sessões com duração de duas horas por doze semanas.

As sessões educativas da pesquisa foram realizadas separadamente para os dois grupos, ou seja, ocorriam em dias diferentes. Após as 12 semanas de intervenção, foram realizadas as reavaliações por meio da aplicação do questionário FSFI, além de duas perguntas que foram feitas às participantes: "Você está satisfeita com a educação que recebeu e ela melhorou suas condições da menopausa?", "Este programa educacional foi eficaz para melhorar suas relações sexuais?". Segundo, Alavi MA, et al. (2016), o programa de aprimoramento sexual melhorou os escores da função sexual, bem como os domínios da excitação e da dor, além de corrigir atitudes em relação ao sexo e aumentar de forma significativa à satisfação e frequência de relações sexuais e visão positiva em relação à vida e aos relacionamentos conjugais.

No artigo de Alavi MA, et al. (2017), também com mulheres na pós-menopausa, a quantidade de grupos foi maior comparado aos demais estudos. No grupo intervenção 1 , as participantes foram submetidas a um programa formal de educação sexual que incorporou materiais sobre vários aspectos desse assunto e envolveu a reformulação das atitudes em relação ao sexo durante a menopausa e a introdução de lubrificantes para aliviar a secura vaginal. No grupo intervenção 2, foi realizado treinamento dos músculos do assoalho pélvico (exercícios de Kegel), ensinados por meio de descrições orais, material escrito, imagens e vídeos, e posteriormente, distribuídas listas de verificação para exercícios diários. Os dois grupos de intervenção foram acompanhados semanalmente por meio de ligações telefônicas.

Ainda nesse estudo, o grupo controle recebeu material educativo geral sobre o climatério. As sessões educacionais presenciais dos 3 grupos foram realizadas com 10 a 15 membros, por 2 horas, usando apresentações e vídeos em PowerPoint, além de receberem livretos educacionais que correspondiam às sessões de que estavam participando. Conforme Alavi MA, et al. (2017), o método de reavaliação foi o mesmo utilizado no estudo citado anteriormente. Não houve diferença significativa entre os 3 grupos em seus escores de função sexual (escores totais e seus domínios),

A pesquisa de Firouzi A, et al. (2019), foi realizada com mulheres na pós-menopausa, a fim de investigar o efeito de um programa educacional na melhora da função sexual feminina. O grupo intervenção recebeu um programa educacional de aconselhamento baseado em parteiras sobre menopausa, alterações 
fisiológicas e anatômicas da menopausa, função e disfunção sexual que afetam durante essa fase, mitos em relação ao sexo, além da participação do cônjuge no processo de avaliação da função sexual erétil. 0 grupo controle recebeu apenas cuidado de rotina para meia-idade, diabetes, pressão arterial, dislipidemia e rastreamento do câncer cervical e de mama. O programa foi executado em 4 sessões realizadas em intervalos de 10 dias, com cada sessão durando 70 minutos.

Os resultados obtidos na literatura de Firouzi A, et al. (2019), identificaram que não houve diferenças significativas entre os dois grupos em termos de características pessoais, sociodemográficos e função sexual antes da intervenção. Após o programa, no grupo intervenção houve melhora significativa no escore total da função sexual e todos seus domínios, no entanto, no grupo controle a diferença não foi relevante.

Os autores Mahnaz E, et al. (2020) efetuaram sua pesquisa com mulheres durante o período gestacional, objetivando analisar o efeito de um pacote educacional estruturado baseado em Análise, Design, Desenvolvimento, Implementação e Avaliação (ADDIE) para reduzir a disfunção sexual durante a gravidez. O modelo ADDIE inclui cinco etapas: análise (coleta de dados, verificação das necessidades, revisões, conteúdo e tarefa das gestantes), design (definição de metas e objetivos), desenvolvimento (ferramentas e processos usados para criar materiais educacionais), implementação (execução dos materiais educativos) e avaliação (análise dos resultados).

As participantes do estudo responderam o questionário FSFI para então serem alocadas, aleatoriamente, em um determinado grupo. Durante as sessões eram fornecidas às gestantes fotos, pôsteres, livretos educativos sobre mudanças fisiológicas durante a gravidez e saúde sexual de modo geral. De acordo com Mahnaz E, et al. (2020), o grupo intervenção recebeu pacote educacional estruturado baseado na função sexual e o grupo controle recebeu treinamento sobre amamentação e parto normal. A intervenção foi realizada durante 4 semanas e foi constatado o aumento da pontuação total do FSFI, bem como as pontuações dos domínios desejo, excitação, lubrificação, orgasmo e satisfação. No entanto, no domínio dor sexual não houve diferença após a intervenção.

Em todos os estudos analisados, as estratégias educativas voltadas para a Função Sexual de mulheres foram desenvolvidas por profissionais de saúde de forma multidisciplinar, enfatizando uma maior diversidade de saberes, contribuindo para nortear a reflexão da população, além de proporcionar uma assistência integral, tornando os usuários ativos no que diz respeito à saúde e qualidade de vida (AGUIAR MIF, et al., 2019). Após a aplicação das práticas educacionais, foi demonstrado melhora do escore total do FSFI e de seus domínios, bem como eliminação de falsas crenças sobre as questões sexuais, aumento da frequência das relações sexuais, atitudes positivas em relação à vida, às relações conjugais e melhora da qualidade de vida de modo geral.

Foram encontradas algumas limitações nesta revisão, tais como um número reduzido de artigos voltados para o tema proposto e delineamento adequado sobre a metodologia da pesquisa. Ainda assim, observouse que a Função Sexual tem um grande impacto na vida de mulheres durante a gravidez, na pósmenopausa e após histerectomia. Diante disso, precisam ser considerados programas educacionais abrangendo a saúde sexual, a fim de disseminar informações e esclarecer possíveis dúvidas, para melhor promoção da saúde.

\section{CONSIDERAÇÕES FINAIS}

Os resultados desta revisão sistemática indicam que a abordagem de ações de Educação em Saúde voltadas para Função Sexual de mulheres melhorou significativamente o funcionamento sexual, independentemente da característica e/ou fase da vida que a mulher esteja vivenciando, além disso, a implementação de programas educacionais promove a melhora da qualidade de vida, autoconhecimento, aumento da autoconfiança e autoestima, podendo levar a relações sexuais mais agradáveis. Além da prevenção de conflitos conjugais, promovendo, assim, a tomada de decisões nos níveis pessoal e interpessoal. É importante sempre levar em consideração o contexto sociocultural, a fim de prestar cuidados em relação à saúde sexual de forma efetiva, respeitando sua individualidade. 


\section{REFERÊNCIAS}

1. ABDO CHN, et al. Tratamento das disfunções sexuais no consultório do ginecologista. Revista Femina, 2019; 47(2): 66-74.

2. AGUIAR MIF. et al. Percepção da equipe multiprofissional da atenção primária sobre educação em saúde. Revista Brasileira de Enfermagem, 2019; 72(1): 278-285.

3. ALAMDARI A, et al. Application of the precede model to improve sexual function among women with hysterectomy. International Journal of Gynecology \& Obstetrics, 2016; 132(2): 229-233.

4. ALAVI MAJD H, et al. Effects of sex education and kegel exercises on the sexual function of postmenopausal women: a randomized clinical trial. The journal of sexual medicine, 2017; 14(7): 959-967.

5. ALAVI MAJD H, et al. The impact of a sexual enhancement program on the sexual function of postmenopausal women. Climacteric, 2016; 19(5): 506-511.

6. AMARAL WN, et al. Função sexual feminina: aspectos normais e patológicos, prevalência no brasil, diagnóstico e tratamento. Revista Femina, 2012; 40(4): 195-202.

7. AMERICAN PSYCHIATRIC ASSOCIATION. Manual Diagnóstico e Estatístico de Transtornos Mentais. Porto Alegre: Artmed, 2002; 4. ed.

8. ARAÚJO JSA, et al. Protocolo Clínico. Avaliação da sexualidade. Hospitais Universitários Federais, 2018.

9. ARAÚJO AC, NETO FL. A nova classificação americana para os transtornos mentais: o DSM-5. Revista brasileira de terapia comportamental e cognitiva, 2014; 16(1): 67-82.

10. BARACHO E. Fisioterapia aplicada à saúde da mulher. 6ª edição. Guanabara Koogan, 2018.

11. BRASIL. Ministério da Saúde. Saúde sexual e saúde reprodutiva: os homens como sujeitos de cuidado, 2018.

12. BRASIL. Ministério da Saúde. Saúde sexual e saúde reprodutiva, 2013.

13. CASTRO MR, et al. Menopausa: marco biopsicossocial do envelhecimento feminino. Psicologia e Sociedade, 2013; 25(2): 410-419.

14. COMERT N, et al. Effects of perineal trauma on postpartum sexual function. journal of advanced nursing,2010; 66(12): 2640-2649.

15. FALKENBERG MB, et al. Educação em saúde e educação na saúde: conceitos e implicações para a saúde coletiva. Ciência \& Saúde Coletiva, 2014; 19: 847-852.

16. FIROUZI A, et al. The effect of a midwife-based counseling education program on sexual function in postmenopausal women: a randomized controlled clinical trial. Menopause, 2019; 26(5): 520-530.

17. GOMES NMC, et al. As práticas de educação em saúde na estratégia saúde da família. Gepnews,2019; 2(2): 99106.

18. KAPLAN HS. A nova terapia do sexo. Editora Nova Fronteira, 1977.

19. MAHNAZ E, et al. Effect of a structured educational package on women's sexual function during pregnancy. International Journal of Gynecology \& Obstetrics, 2020; 148(2): 225-230.

20. MASTERS WH, JOHNSON VE. A resposta sexual humana. Editora Roca, 1984.

21. MOHER D, et al. Preferred reporting items for systematic reviews and meta-analyses: the PRISMA statement. Annals of internal medicine, 2009; 151(4): 264-269.

22. VRABEL M. Preferred reporting items for systematic reviews and meta analyses. In: Oncology nursing fórum. Oncology Nursing Society, 2015; 55. 